\title{
The $\tau$ Magnetic Dipole Moment at Future Lepton Colliders
}

\author{
Jessica N. Howard, ${ }^{1}$ Arvind Rajaraman, ${ }^{1}$ Rebecca Riley, ${ }^{1}$ and Tim M. P. Tait ${ }^{1}$ \\ ${ }^{1}$ Department of Physics and Astronomy, University of California, Irvine, CA 92697, USA \\ Received: 11 Dec 2018, Accepted: 25 Dec 2018, Published: 1 Apr 2019
}

\begin{abstract}
$\overline{\text { Abstract }}$
The magnetic moment of the $\tau$ lepton is an interesting quantity that is potentially sensitive to physics beyond the Standard Model. Electroweak gauge invariance implies that a heavy new physics contribution to it takes the form of an operator which involves the Higgs boson, implying that rare Higgs decays are able to probe the same physics as $a_{\tau}$. We examine the prospects for rare Higgs decays at future high energy lepton (electron or muon) colliders, and find that such a project collecting a few ab ${ }^{-1}$ would be able to advance our understanding of this physics by roughly a factor of 10 compared to the expected reach of the high luminosity LHC.
\end{abstract}

Keywords: symmetry, Lepton Collider, magnetic dipole moment, tau

DOI: 10.31526/LHEP.2.2019.113

\section{INTRODUCTION}

Anomalous magnetic moments of charged fermions occupy a special role in our understanding of the Standard Model (SM) of particle physics. Early on, the tiny electromagnetic correction to the electron's magnetic moment provided one of the first indications that quantum field theory was the correct language to describe subatomic physics. More recently, the anomalous magnetic moment of the muon is one of the few experimental measurements to stubbornly resist being well-described by the Standard Mode ${ }^{1}$ [7]. A short distance contribution to the anomalous magnetic moment takes the form of a dimension-5 operator,

$$
a_{\psi} \frac{e}{2 m_{\psi}} \bar{\psi} \sigma^{\mu v} \psi F_{\mu v}
$$

which implies a chiral-flip on the fermion. Since chirality change involves an insertion of the mass, it is natural to speculate that if there is new physics subtly influencing the muon magnetic moment, it should manifest even more strongly for the tau lepton.

The electroweak $S U(2) \times U(1)$ gauge symmetry would forbid chirality-changing interactions of the SM fermions. Consequently, their presence ultimately derives from the fact that the symmetry is spontaneously broken by the vacuum expectation value (VEV) of the Higgs. Indeed, promoting the tau magnetic moment operator into an $S U(2) \times U(1)$ invariant form leads to a pair of dimension six terms,

$$
c_{1} \bar{\tau}_{R} \sigma^{\mu v} B_{\mu \nu} H^{\dagger} L_{3}+c_{2} \bar{\tau}_{R} \sigma^{\mu v} H^{\dagger} W_{\mu v} L_{3}+\text { h.c. }
$$

where $L_{3}$ is the left-handed $S U(2)$ lepton doublet containing $\tau_{L}, H$ is the Higgs doublet, $B_{\mu \nu}$ and $W_{\mu v}$ are the field strengths for the hypercharge and $S U(2)$ gauge bosons, and $c_{1}$ and $c_{2}$ are (generically complex) coefficients with units of (energy) $)^{-2}$ which encapsulate the residual effects of heavy physics at low

\footnotetext{
${ }^{1}$ It also bears mentioning that puzzling results for the proton radius extracted from muonic hydrogen [1] and indications for lepton-non-universality in semileptonic $B$ decays [2] 3 4 4 5] 6 may further point to new physics influencing muonic observables.
}

energies. Replacing the Higgs with its VEV generates the magnetic dipole moment (and also generically modify $Z$ boson couplings and, if $c_{1}$ and $c_{2}$ are complex, contributes to the electric dipole). But such interactions necessarily imply a modification of the coupling of the Higgs to $\tau^{+} \tau^{-} \gamma$ as well,

$$
\frac{1}{\Lambda^{2}} h \bar{\tau} \sigma^{\mu \nu} \tau F_{\mu \nu}
$$

where $h$ is the field corresponding to the Higgs boson and $\Lambda$ is a linear combination of the original couplings $c_{1}$ and $c_{2}$. Thus, rare Higgs decays offer a complementary, high energy probe of anomalous magnetic moments. Guided by the expectation that new physics may be more evident for the tau, we focus on the operator described in Equation (3).

Our current understanding of the tau magnetic dipole moment is relatively modest. Previous experiments have focused on precision measurements of the $\tau$ itself $[8,9,10,11,12,13,14$ 15, 16. 17, 18, 19, 20, 21, 22, 23, with the strongest constraints on these operators coming from the kinematics of the production process $e^{+} e^{-} \rightarrow \tau^{+} \tau^{-}$at LEP2 [24, 25], which places the limit:

$$
-0.052<a_{\tau}^{\gamma}<0.013, \quad 95 \% \text { CL, }
$$

corresponding to [26]:

$$
|\Lambda|>\begin{aligned}
& 333 \mathrm{GeV} \\
& 666 \mathrm{GeV}
\end{aligned} \quad \text { for : } \begin{aligned}
& \Lambda^{2}>0 \\
& \Lambda^{2}<0
\end{aligned} .
$$

At higher energies, future lepton colliders can directly produce the Higgs boson, and look for the rare Higgs decay

$$
h \rightarrow \tau^{+} \tau^{-} \gamma
$$

in which the new physics contribution interferes with the SM decay $h \rightarrow \tau^{+} \tau^{-}$followed by radiation of a hard photon from one of the $\tau^{\prime}$ s. Constraints on the size of this rare decay imply bounds on the size of $\Lambda$, and hence on the magnetic moment. This process was previously studied in Ref. [26], and found to be promising for the end stage LHC running in a high luminosity mode. In this work, we extend this study to future lepton colliders and examine the contribution such machines could contribute to our understanding of the tau magnetic moment. 


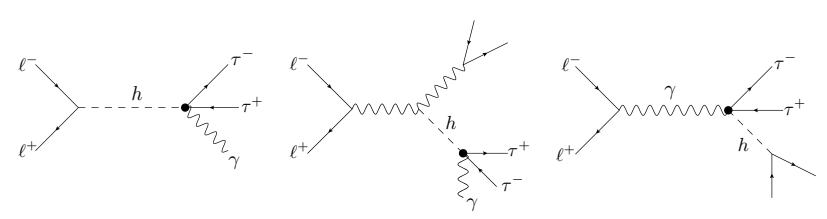

FIGURE 1: Representative Feynman diagrams for the processes $\mu^{+} \mu^{-} \rightarrow h^{(*)} \rightarrow \tau^{+} \tau^{-} \gamma, \ell^{+} \ell^{-} \rightarrow Z^{*} \rightarrow Z h$ followed by $h \rightarrow$ $\tau^{+} \tau^{-} \gamma$, and $\ell^{+} \ell^{-} \rightarrow \gamma^{*} \rightarrow \tau^{+} \tau^{-} h$ (left to right).

\section{FUTURE LEPTON COLLIDERS}

The operator of Equation (3) leads to exotic processes involving a Higgs boson, a photon, and a $\tau^{+} \tau^{-}$pair. At a future high energy lepton collider, there are a number of potential ways to search for its presence. We consider three reactions which can probe this process:

- $\mu^{+} \mu^{-} \rightarrow h^{(*)} \rightarrow \tau^{+} \tau^{-} \gamma$

- $\ell^{+} \ell^{-} \rightarrow Z^{*} \rightarrow Z h$ followed by $h \rightarrow \tau^{+} \tau^{-} \gamma$; or

- $\ell^{+} \ell^{-} \rightarrow \gamma^{*} \rightarrow \tau^{+} \tau^{-} h$ followed by any SM Higgs decay.

Representative Feynman diagrams for the three processes are shown in Figure 1. The first two processes probe the $\tau$ magnetic dipole moment via Higgs decay, and thus typically have momentum transfer characterized by $m_{h}$. The third process involves a momentum transfer of order $\sqrt{s}$, which may be important at very high energies. In these reactions $\ell \equiv e, \mu$ are relevant both at future electron and future muon colliders, whereas the first process is very suppressed at an $e^{+} e^{-}$machine due to the tiny electron Yukawa interaction.

\section{1. $\ell^{+} \ell^{-} \rightarrow Z^{*} \rightarrow \mathrm{Zh}$}

We find that for intermediate collider energy, the process $\ell^{+} \ell^{-} \rightarrow Z^{*} \rightarrow Z h$ provides the most stringent constraint, with $\ell^{+} \ell^{-} \rightarrow \gamma^{*} \rightarrow \tau^{+} \tau^{-} h$ becoming comparable at very high collider energies. For the energies considered, the process $\mu^{+} \mu^{-} \rightarrow h^{(*)} \rightarrow \tau^{+} \tau^{-} \gamma$ always involves an off-shell Higgs boson, and is never the dominant probe. We therefore focus our discussion on the $Z h$ production mode, followed by $h \rightarrow \tau^{+} \tau^{-} \gamma$, leading to a $Z \tau^{+} \tau^{-} \gamma$ final state.

The detailed experimental reconstruction depends rather sensitively on the design and performance of the detectors, and are not currently completely well-defined. However, all current plans propose very precise detectors providing exquisite information via calorimetric and tracking metrics, which are likely to reconstruct all visible particles produced in a collision. For this reason, we we eschew a specific detector simulation and instead work at the parton level, imposing stiff reconstruction cuts such that we expect the $\tau^{\prime}$ s, $\gamma^{\prime}$ s, and $Z$ bosons (for visible decays) can be reconstructed with near-perfect precision,

$$
p_{T}^{\gamma, \tau} \geq 10 \mathrm{GeV}, \quad\left|\eta^{\gamma, \tau}\right| \leq 2.5,
$$

where $p_{T}$ and $\eta$ are the transverse momentum and pseudorapidity, respectively. Specifically, these stiff cuts on the $\tau$ s insure that their decay products are collimated which allows their momenta to be reconstructed reasonably accurately, despite the unmeasured energy going into neutrinos (for a more detailed discussion of the requirements in a similar context, see Ref.
[27]). We simulate both signal and SM background processes using MadGraph5_aMC@NLO (MG5) [28], with the FeynRules SM implementation [29, 30, 31], supplemented with the tau dipole operators of Eq. 2].

\subsubsection{Background and Selection Cuts}

There are multiple SM background processes contributing to the $Z \tau^{+} \tau^{-} \gamma$ signature. These are primarily:

- radiation of a photon from the initial state of $\ell^{+} \ell^{-} \rightarrow Z Z$ followed by one of the $Z$ bosons decaying into $\tau^{+} \tau^{-}$;

- initial state photon radiation from $\ell^{+} \ell^{-} \rightarrow Z h$, where $h \rightarrow \tau^{+} \tau^{-}$; and

- final state radiation of a photon from a $\tau$ in the process $\ell^{+} \ell^{-} \rightarrow Z Z$ where one $Z$ decays to $\tau^{+} \tau^{-} \gamma$

The initial state radiation contributions to the background are slightly different for electron and muon beams, because of the dependence on the lepton mass in the collinear region of kinematics.

The primary tool to sift the new physics signal from these backgrounds is to reconstruct the invariant masses $M_{\tau \tau}$ and $M_{\tau \tau \gamma}$. In order to remove backgrounds where the $\tau$ s are produced by off-shell photons or close to on-shell $Z$ bosons, we exclude events for which,

$$
M_{\tau \tau} \leq 10 \mathrm{GeV} \quad \text { and } \quad 80 \mathrm{GeV} \leq M_{\tau \tau} \leq 100 \mathrm{GeV}
$$

We would also like to avoid processes for which the $\tau$ s are produced by an on-shell Higgs boson decay, with the additional photon radiated from the initial state. This is accomplished by vetoing events which satisfy,

$$
120 \mathrm{GeV} \leq M_{\tau \tau} \leq 130 \mathrm{GeV}
$$

We further select events for which the $\tau \tau \gamma$ are consistent with coming from an on-shell Higgs decay, requiring,

$$
120 \mathrm{GeV} \leq M_{\tau \tau \gamma} \leq 130 \mathrm{GeV}
$$

Our choice of cuts is conservative in the sense that they assume windows around the $Z$ and Higgs masses which are $\mathcal{O}(10 \%)$, far larger than the expected order percent level experimental resolution for a realistic detector or the intrinsic widths of the $Z$ and Higgs bosons. In Table 1, we show the cross sections before and after cuts for simulations containing the SM alone, and for the SM plus the new physics operator for two choices of

$$
\alpha \equiv \frac{G_{F}}{\Lambda^{2}}
$$

$\alpha=0.1$, and $\alpha=0.2$ for the representative case of an $e^{+} e^{-}$ collider operating at a center-of-mass energy $\sqrt{s}=500 \mathrm{GeV}$.

\subsubsection{Analysis}

We extract the sensitivity to the $\tau$ magnetic dipole operator by writing the amplitude for the signal process with the dependence on $\alpha$ explicitly factored out,

$$
\mathcal{M}_{\text {sig }}=\mathcal{M}_{\mathrm{SM}}+\alpha \mathcal{M}_{\mathrm{NP}}
$$




\begin{tabular}{c|c|c|c} 
& $\mathrm{SM}$ & $\alpha=0.1$ & $\alpha=0.2$ \\
\hline Before cuts & 1.90 & 2.55 & 4.43 \\
$M_{\tau \tau}$ cuts & 0.666 & 0.898 & 1.60 \\
$M_{\tau \tau \gamma}$ cuts & 0.00334 & 0.130 & 0.455
\end{tabular}

TABLE 1: Cross sections (fb) before and after cuts for the process $e^{+} e^{-} \rightarrow Z \tau^{+} \tau^{-} \gamma$ at $\sqrt{s}=500 \mathrm{GeV}$ in the SM and for two choices of $\alpha$, as indicated.

\begin{tabular}{c|c|c|c}
$\sqrt{s}$ & $\alpha=0.001$ & $\alpha=0.045$ & $\alpha=0.1$ \\
\hline $250 \mathrm{GeV}$ & $7.24 \times 10^{6}$ & $3.28 \times 10^{3}$ & $6.36 \times 10^{2}$ \\
$500 \mathrm{GeV}$ & $2.66 \times 10^{7}$ & $5.51 \times 10^{3}$ & $3.51 \times 10^{2}$ \\
$600 \mathrm{GeV}$ & $1.53 \times 10^{6}$ & $2.66 \times 10^{4}$ & $3.08 \times 10^{2}$ \\
$800 \mathrm{GeV}$ & $7.35 \times 10^{5}$ & $4.57 \times 10^{3}$ & $2.31 \times 10^{2}$ \\
$1000 \mathrm{GeV}$ & $3.71 \times 10^{5}$ & $3.04 \times 10^{3}$ & $2.18 \times 10^{2}$
\end{tabular}

TABLE 2: Integrated luminosity (in $\mathrm{fb}^{-1}$ ) required for a $5 \sigma$ deviation for different center of mass energies and values of $\alpha$ at an $e^{+} e^{-}$collider.

\begin{tabular}{c|c|c|c}
$\sqrt{s}$ & $\alpha=0.001$ & $\alpha=0.045$ & $\alpha=0.1$ \\
\hline $250 \mathrm{GeV}$ & $8.63 \times 10^{6}$ & $3.98 \times 10^{3}$ & $7.75 \times 10^{2}$ \\
$500 \mathrm{GeV}$ & $2.92 \times 10^{6}$ & $2.60 \times 10^{5}$ & $4.59 \times 10^{2}$ \\
$600 \mathrm{GeV}$ & $1.23 \times 10^{6}$ & $2.40 \times 10^{5}$ & $3.45 \times 10^{2}$ \\
$800 \mathrm{GeV}$ & $4.22 \times 10^{5}$ & $2.94 \times 10^{4}$ & $2.48 \times 10^{2}$ \\
$1000 \mathrm{GeV}$ & $8.04 \times 10^{4}$ & $1.56 \times 10^{4}$ & $2.46 \times 10^{2}$
\end{tabular}

TABLE 3: Integrated luminosity (in $\mathrm{fb}^{-1}$ ) required for a $5 \sigma$ deviation for different center of mass energies and values of $\alpha$ at a $\mu^{+} \mu^{-}$collider.

for which the cross-section (after cuts) for a specific $\sqrt{s}$ takes the form

$$
\sigma(\alpha)=\sigma_{\mathrm{SM}}+2 \alpha \sigma_{\mathrm{INT}}+\alpha^{2} \sigma_{\mathrm{NP}}
$$

where $\sigma_{\mathrm{SM}}, \sigma_{\mathrm{INT}}$, and $\sigma_{\mathrm{NP}}$ represent the $\mathrm{SM}$ cross section, interference term, new physics contributions, respectively. They are extracted for $\sqrt{s}=500 \mathrm{GeV}$ from the bottom row of Table 1 . and likewise for other energies simulated.

From here, we determine the required luminosity such that the difference between the number of events predicted for the Standard Model $B$ and the total number of expected events for a given $\alpha, S+B$ corresponds to a $5 \sigma$ statistically significant deviation, $\frac{S}{\sqrt{S+B}}>5$ in the regime of Gaussian statistics, $S+B>5$. Tables 2 and 3 show the required luminosity in $\mathrm{fb}^{-1}$ for a $5 \sigma$ deviation at an $e^{+} e^{-}$and $\mu^{+} \mu^{-}$collider, for various energies and values of $\alpha$. The same results are presented graphically in Figure 2 Evident from the tables, values of $\Lambda$ of order a few $\mathrm{TeV}$ can be probed with datasets of order hundreds of $\mathrm{fb}^{-1}$ for $\sqrt{s} \gtrsim 500 \mathrm{GeV}$, whereas reaching $\Lambda$ of order $10 \mathrm{~s}$ of $\mathrm{TeV}$ requires a few $\mathrm{ab}^{-1}$ and $\sqrt{s} \gtrsim 800 \mathrm{GeV}$.

\section{2. $\ell^{+} \ell^{-} \rightarrow \gamma^{*} \rightarrow \tau^{+} \tau^{-} h$}

At higher collider energies, the process $\ell^{+} \ell^{-} \rightarrow \gamma^{*} \rightarrow \tau^{+} \tau^{-} h$ can take advantage of the larger $s$-channel momentum transfer, resulting in a larger lever arm when studying short distance physics. We simulate this process, assuming that the Higgs can be reconstructed with near-perfect efficiency regardless of its decay mode, and apply selection cuts to the $p_{T}$ and $\eta$ of the

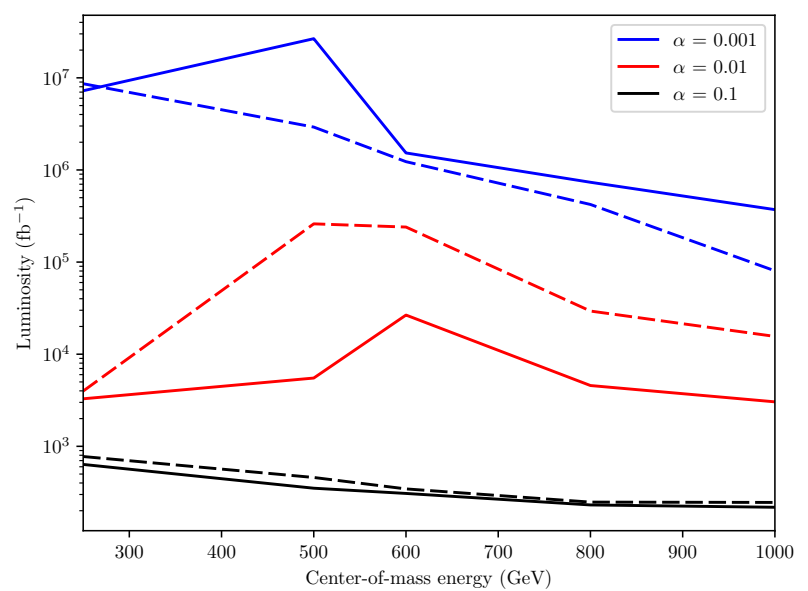

FIGURE 2: Integrated luminosity required for a $5 \sigma$ discovery as a function of center of mass energy at a future $e^{+} e^{-}$(solid lines) or $\mu^{+} \mu^{-}$(dashed lines) collider for the indicated values of $\alpha$.

\begin{tabular}{c|c|c|c}
$\sqrt{s}$ & $\alpha=0.001$ & $\alpha=0.045$ & $\alpha=0.1$ \\
\hline $1 \mathrm{TeV}$ & $1.05 \times 10^{5}$ & $5.37 \times 10^{1}$ & $1.09 \times 10^{1}$ \\
$5 \mathrm{TeV}$ & $1.90 \times 10^{3}$ & $9.36 \times 10^{-1}$ & $1.90 \times 10^{-1}$ \\
$10 \mathrm{TeV}$ & $4.72 \times 10^{2}$ & $2.33 \times 10^{-1}$ & $4.72 \times 10^{-2}$
\end{tabular}

TABLE 4: Integrated luminosity (in $\mathrm{fb}^{-1}$ ) required for a $5 \sigma$ deviation for different center of mass energies and values of $\alpha$ at an $e^{+} e^{-}$collider.

\begin{tabular}{c|c|c|c}
$\sqrt{s}$ & $\alpha=0.001$ & $\alpha=0.045$ & $\alpha=0.1$ \\
\hline $1 \mathrm{TeV}$ & $2.19 \times 10^{5}$ & $2.44 \times 10^{2}$ & $5.08 \times 10^{1}$ \\
$5 \mathrm{TeV}$ & $1.91 \times 10^{3}$ & $9.42 \times 10^{-1}$ & $1.91 \times 10^{-1}$ \\
$10 \mathrm{TeV}$ & $4.69 \times 10^{2}$ & $2.32 \times 10^{-1}$ & $4.69 \times 10^{-2}$
\end{tabular}

TABLE 5: Integrated luminosity (in $\mathrm{fb}^{-1}$ ) required for a $5 \sigma$ deviation for different center of mass energies and values of $\alpha$ at an $\mu^{+} \mu^{-}$collider.

tau leptons and photons as before. We select high momentum transfer by retaining events for which

$$
M_{\tau \tau} \geq 100 \mathrm{GeV}
$$

Using the same criteria as before, we determine the required integrated luminosity for a $5 \sigma$ deviation for a variety of center of mass energies and coupling strengths $\alpha$. In Tables 4 and 5 . we show the results for $e^{+} e^{-}$and $\mu^{+} \mu^{-}$colliders, respectively. These results indicate that an $\mathcal{O}(10 \mathrm{TeV})$ lepton collider would provide a very effective probe of new physics relevant for the tau magnetic dipole moment.

\section{CONCLUSIONS AND OUTLOOK}

Given the longstanding discrepancy between the measurement of the anomalous magnetic moment of the muon and its SM predictions, it is natural to wonder if $a_{\tau}$ might show a related discrepancy enhanced by its larger mass. The electroweakviolating nature of the operators contributing to $a_{\tau}$ imply that 
an indirect way to access it is through rare Higgs decays into $\tau^{+} \tau^{-} \gamma$

We have examined the prospects for such a measurement at a future high energy lepton collider, simulating signal and SM backgrounds (including the important interference between the two) at the parton level. We find that at $\sqrt{s} \gtrsim 800 \mathrm{GeV}$ and having collected a few $a b^{-1}$ of integrated luminosity, it is likely that such a machine can probe this operator to about a factor of 10 better than the expectations at the high luminosity LHC. Given these initial promising results, it would be worthwhile to follow up this study with one based on more realistic detector simulations.

\section{ACKNOWLEDGMENTS}

The work of AR and TMPT is supported in part by National Science Foundation grant PHY-1620638. The code used for selection cuts and various other aspects of this analysis is located at https://github.com/rebecca-riley/muon_collider.

\section{References}

[1] R. Pohl et al., "The size of the proton," Nature 466, 213 (2010).

[2] R. Aaij et al. [LHCb Collaboration], "Differential branching fractions and isospin asymmetries of $B \rightarrow K^{(*)} \mu^{+} \mu^{-}$ decays," JHEP 1406, 133 (2014) [arXiv:1403.8044 [hep-ex]].

[3] R. Aaij et al. [LHCb Collaboration], "Angular analysis and differential branching fraction of the decay $B_{S}^{0} \rightarrow$ $\phi \mu^{+} \mu^{-}$, , JHEP 1509, 179 (2015) [arXiv:1506.08777 [hepex]].

[4] R. Aaij et al. [LHCb Collaboration], "Angular analysis of the $B^{0} \rightarrow K^{* 0} \mu^{+} \mu^{-}$decay using $3 \mathrm{fb}^{-1}$ of integrated luminosity," JHEP 1602, 104 (2016) [arXiv:1512.04442 [hep-ex]].

[5] R. Aaij et al. [LHCb Collaboration], "Measurements of the S-wave fraction in $B^{0} \rightarrow K^{+} \pi^{-} \mu^{+} \mu^{-}$decays and the $B^{0} \rightarrow K^{*}(892)^{0} \mu^{+} \mu^{-}$differential branching fraction," JHEP 1611, 047 (2016) Erratum: [JHEP 1704, 142 (2017)] [arXiv:1606.04731 [hep-ex]].

[6] S. Wehle et al. [Belle Collaboration], "Lepton-FlavorDependent Angular Analysis of $B \rightarrow K^{*} \ell^{+} \ell^{-}$, , Phys. Rev. Lett. 118, no. 11, 111801 (2017) [arXiv:1612.05014 [hep-ex]].

[7] G. W. Bennett et al. [Muon g-2 Collaboration], "Final Report of the Muon E821 Anomalous Magnetic Moment Measurement at BNL," Phys. Rev. D 73, 072003 (2006) [hep-ex/0602035].

[8] D. J. Silverman and G. L. Shaw, "Limits on the Composite Structure of the Tau Lepton and Quarks From Anomalous Magnetic Moment Measurements in $e^{+} e^{-}$Annihilation," Phys. Rev. D 27, 1196 (1983).

[9] L. D. Almeida, A. A. Natale, S. F. Novaes and O. J. P. Eboli, "Nonstandard gamma gamma $\rightarrow$ lepton+ lepton- processes in relativistic heavy ion collisions," Phys. Rev. D 44, 118 (1991).

[10] F. del Aguila, F. Cornet and J. I. Illana, "The Possibility of using a large heavy ion collider for measuring the electromagnetic properties of the tau-lepton," Phys. Lett. B 271, 256 (1991).

[11] M. A. Samuel and G. Li, "Measuring the magnetic moment of the tau lepton at the Fermilab tevatron, the, SSC and the LHC," Int. J. Theor. Phys. 33, 1471 (1994).
[12] A. Aeppli and A. Soni, "Analysis of the tau dipole moment via e+ e- and gamma gamma reactions," Phys. Rev. D 46, 315 (1992).

[13] R. Escribano and E. Masso, "New bounds on the magnetic and electric moments of the tau lepton," Phys. Lett. B 301, 419 (1993).

[14] R. Escribano and E. Masso, "Improved bounds on the electromagnetic dipole moments of the $\tau$ lepton," Phys. Lett. B 395, 369 (1997) [hep-ph/9609423].

[15] G. A. Gonzalez-Sprinberg, A. Santamaria and J. Vidal, "Model independent bounds on the tau lepton electromagnetic and weak magnetic moments," Nucl. Phys. B 582, 3 (2000) [hep-ph/0002203].

[16] J. Bernabeu, G. A. Gonzalez-Sprinberg, J. Papavassiliou and J. Vidal, "Tau anomalous magnetic moment formfactor at super B/flavor factories," Nucl. Phys. B 790, 160 (2008) [arXiv:0707.2496 [hep-ph]].

[17] J. Bernabeu, G. A. Gonzalez-Sprinberg and J. Vidal, "Tau spin correlations and the anomalous magnetic moment," JHEP 0901, 062 (2009) [arXiv:0807.2366 [hep-ph]].

[18] S. Atag and A. A. Billur, "Possibility of Determining $\tau$ Lepton Electromagnetic Moments in $\gamma \gamma \rightarrow \tau^{+} \tau^{-}$ Process at the CERN-LHC," JHEP 1011, 060 (2010) [arXiv:1005.2841 [hep-ph]].

[19] J. Peressutti and O. A. Sampayo, " $\tau$ anomalous magnetic moment in $\gamma \gamma$ colliders," Phys. Rev. D 86, 035016 (2012).

[20] A. Hayreter and G. Valencia, "Constraining $\tau$-lepton dipole moments and gluon couplings at the LHC," Phys. Rev. D 88, no. 1, 013015 (2013) Erratum: [Phys. Rev. D 91, no. 9, 099902 (2015)] [arXiv:1305.6833 [hep-ph]].

[21] M. Fael, L. Mercolli and M. Passera, "Towards a determination of the tau lepton dipole moments," Nucl. Phys. Proc. Suppl. 253-255, 103 (2014) [arXiv:1301.5302 [hep$\mathrm{ph}]$.

[22] A. Hayreter and G. Valencia, "Spin correlations and new physics in $\tau$-lepton decays at the LHC," JHEP 1507, 174 (2015) [arXiv:1505.02176 [hep-ph]].

[23] S. Eidelman, D. Epifanov, M. Fael, L. Mercolli and M. Passera, " $\tau$ dipole moments via radiative leptonic $\tau$ decays," JHEP 1603, 140 (2016) [arXiv:1601.07987 [hep-ph]].

[24] J. Abdallah et al. [DELPHI Collaboration], "Study of tau-pair production in photon-photon collisions at LEP and limits on the anomalous electromagnetic moments of the tau lepton," Eur. Phys. J. C 35, 159 (2004) [hepex/0406010].

[25] A. Heister et al. [ALEPH Collaboration], "Search for anomalous weak dipole moments of the tau lepton," Eur. Phys. J. C 30, 291 (2003) [hep-ex/0209066].

[26] I. Galon, A. Rajaraman and T. M. P. Tait, JHEP 1612, 111 (2016) doi:10.1007/JHEP12(2016)111 [arXiv:1610.01601 [hep-ph]].

[27] B. Murakami and T. M. P. Tait, Phys. Rev. D 91, 015002 (2015) doi:10.1103/PhysRevD.91.015002 [arXiv:1410.1485 [hep-ph]].

[28] J. Alwall et al., JHEP 1407, 079 (2014) doi:10.1007/JHEP07(2014)079 [arXiv:1405.0301 [hep$\mathrm{ph}]$.

[29] C. Degrande, C. Duhr, B. Fuks, D. Grellscheid, O. Mattelaer and T. Reiter, Comput. Phys. Commun. 183, 1201 (2012) doi:10.1016/j.cpc.2012.01.022 [arXiv:1108.2040 [hep-ph]]. 
[30] A. Alloul, N. D. Christensen, C. Degrande, C. Duhr and B. Fuks, Comput. Phys. Commun. 185, 2250 (2014) doi:10.1016/j.cpc.2014.04.012 [arXiv:1310.1921 [hep-ph]].

[31] A. Alloul, B. Fuks and V. Sanz, JHEP 1404, 110 (2014) doi:10.1007/JHEP04(2014)110 [arXiv:1310.5150 [hep-ph]]. 